\title{
ON PROBABILTY DISTRIBUTION IN ASSOCIATION WITH A CERTAIN GENERALIZED HYPERGEOMETRIC FUNCTION
}

\author{
Yashwant Singh \\ Department of Mathematics, \\ Government College, Kaladera, Jaipur, Rajasthan, India \\ E-mail: dryashu23@yahoo.in \\ And \\ Nanda Kulkarni $\left(^{*}\right)$ \\ Department of Mathematics, \\ Maharani Lakshmi Ammanni College for Women Bangalore \\ Karnataka, India \\ E-mail: nandavnadiger@gmail.com \\ $\left.{ }^{*}\right)$ Research Scholar: Department of Mathematics, \\ J.J.T.University, Chudela, Jhunjhunu (Rajasthan), India
}

\begin{abstract}
In the present paper, a probability function $P(x)$ has been introduced in terms of the $\bar{H}$-function and its properties are studied. It is shown that the classical non-central distributions such as, non-central chi-square, non-central Student- $t$, non-central $F$ and almost all classical central continuous distributions can be obtained as special cases of this general density function. This general density function $P(x)$ is introduced with the hope that any density function, which can be represented in terms of any known special function as well as the density of the ratio of any two independent stochastic variables whose density functions can be represented in terms of any known special functions, is contained in $P(x)$ as a special case. The properties of $P(x)$, discussed in this paper, include the characteristic function, moments, recurrence relationship among moments and the distribution function.
\end{abstract}

Ker words: Non-central Distribution, $\bar{H}$-function, General Probability Function, Ratio Distribution. (2000 Mathematics subject classification: 33C99)

\section{Council for Innovative Research}

Peer Review Research Publishing System

Journal: INTERNATIONAL JOURNAL OF COMPUTERS \& TECHNOLOGY

Vol 14 . No. 3

www.ijctonline.com, editorijctonline@gmail.com 


\section{Introduction}

The $\bar{H}$-function occurring in the paper will be defined and represented by Inayat-Hussain [8] as follows:

$$
\bar{H}_{P, Q}^{M, N}[z]=\bar{H}_{P, Q}^{M, N}\left[z \mid \begin{array}{c}
\left(a_{j} ; \alpha_{j} ; A_{j}\right)_{1, N},\left(a_{j} ; \alpha_{j}\right)_{N+1, P} \\
\left(b_{j}, \beta_{j}\right)_{1, M},\left(b_{j}, \beta_{j} ; B_{j}\right)_{M+1, Q}
\end{array}\right]=\frac{1}{2 \pi i} \int_{-i \infty}^{i \infty} \bar{\phi}(\xi) z^{\xi} d \xi
$$

where $\quad \bar{\phi}(\xi)=\frac{\prod_{j=1}^{M} \Gamma\left(b_{j}-\beta_{j} \xi\right) \prod_{j=1}^{N}\left\{\Gamma\left(1-a_{j}+\alpha_{j} \xi\right)\right\}^{A_{j}}}{\prod_{j=M+1}^{Q}\left\{\Gamma\left(1-b_{j}+\beta_{j} \xi\right)\right\}^{B_{j}} \prod_{j=N+1}^{P} \Gamma\left(a_{j}-\alpha_{j} \xi\right)}$

Which contains fractional powers of the gamma functions. Here, and throughout the paper $a_{j}(j=1, \ldots, p)$ and $b_{j}(j=1, \ldots, Q)$ are complex parameters, $\alpha_{j} \geq 0(j=1, \ldots, P), \beta_{j} \geq 0(j=1, \ldots, Q)$ (not all zero simultaneously) and exponents $A_{j}(j=1, \ldots, N)$ and $B_{j}(j=N+1, \ldots, Q)$ can take on non integer values.

The following sufficient condition for the absolute convergence of the defining integral for the $\bar{H}$-function given by equation (1.1) have been given by (Buschman and Srivastava[2]).

$\Omega \equiv \sum_{j=1}^{M}\left|\beta_{j}\right|+\sum_{j=1}^{N}\left|A_{j} \alpha_{j}\right|-\sum_{j=M+1}^{Q}\left|\beta_{j} B_{j}\right|-\sum_{j=N+1}^{P}\left|\alpha_{j}\right|>0$

and $|\arg (z)|<\frac{1}{2} \pi \Omega$

The behavior of the $\bar{H}$-function for small values of $|z|$ follows easily from a result recently given by (Rathie [13],p.306,eq.(6.9)).

We have

$\bar{H}_{P, Q}^{M, N}[z]=0\left(|z|^{\gamma}\right), \gamma=\min _{1 \leq j \leq N}\left[\operatorname{Re}\left(b_{j} / \beta_{j}\right)\right],|z| \rightarrow 0$

If we take $A_{j}=1(j=1,2, \ldots, N), B_{j}=1(j=M+1, \ldots, Q)$ in (1.1), the function $\bar{H}_{P, Q}^{M, N}[$.$] reduces to the Fox's H$ function [6].

The following series representation for the $\bar{H}$-function will be required in the sequel (see Rathie, [13]pp.305306, eq.(6.8)):

$$
\begin{aligned}
\bar{H}_{P, Q}^{M, N}\left[z \mid \begin{array}{l}
\left(a_{j}, \alpha_{j} ; A_{j}\right)_{1, N}\left(a_{j}, a_{j}\right)_{N+1, P} \\
\left(b_{j}, \beta_{j}\right)_{1, M},\left(b_{j}, \beta_{j} ; B_{j}\right)_{M+1, Q}
\end{array}\right]= & \frac{\sum_{h=1}^{M} \sum_{r=0}^{\infty} \prod_{\substack{j=1 \\
j \neq h}}^{M} \Gamma\left(b_{j}-\beta_{j} \xi_{h, r}\right) \prod_{j=1}^{N}\left\{\Gamma\left(1-a_{j}+\alpha_{j} \xi_{h, r}\right)\right\}^{A_{j}}(-1)^{r} z^{\xi_{h, r}}}{\prod_{j=M+1}^{Q}\left\{\Gamma\left(1-b_{j}+\beta_{j} \xi_{h, r}\right)\right\}^{B_{j}} \prod_{j=N+1}^{P} \Gamma\left(a_{j}-\alpha_{j} \xi_{h, r}\right) r ! \beta_{h}}
\end{aligned}
$$

Where

$\xi_{h, r}=\frac{\left(b_{h}+r\right)}{\beta_{h}}$. 


\section{Some Definitions and Preliminary Results}

Result 1.

$\int_{0}^{\infty} \frac{x^{\lambda-1}}{\left(1+b x^{k}\right)^{\mu-1}} \bar{H}_{p, q}^{m, n}\left[\left.\left(\frac{a x^{k}}{\left(1+b x^{k}\right)}\right)^{s}\right|_{B^{*}} ^{A^{*}}\right] d x=$

$k^{-1} b^{-\frac{\lambda}{k}} \Gamma\left(\mu-1-\frac{\lambda}{k}\right) \bar{H}_{p+1, q+1}^{m, n+1}\left[\left(\frac{a}{b}\right)^{s} \mid\left(\begin{array}{c}\left.1-\frac{\lambda}{k},-s ; 1\right), A^{*} \\ B^{*},(2-\mu,-s ; 1)\end{array}\right]\right.$

Where $\operatorname{Re}\left(\lambda-k s \frac{b_{j}}{\beta_{j}}\right)>0$ for $j=1,2, \ldots, m ; \operatorname{Re}(\lambda-k \mu+k)<0, k>0, b>0$ and $\operatorname{Re}($.$) means the real$ part of (.) .

This result follows easily from the fact that,

$\int_{0}^{\infty} x^{\lambda-1}\left(1+b x^{k}\right)^{\mu-1} d x=\frac{k^{-1} b^{-\frac{\lambda}{k}} \Gamma\left(\frac{\lambda}{k}\right) \Gamma\left(1-\mu-\frac{\lambda}{k}\right)}{\Gamma(1-\mu)}$

Where $b, k>0,0<\operatorname{Re}\left(\frac{\lambda}{k}\right), \operatorname{Re}(1-\mu)$ on employing (1.1).

Result 2

$\int_{0}^{\infty} e^{-d x} \frac{x^{\lambda-1}}{\left(1+b x^{k}\right)^{\mu-1}} \bar{H}_{p, q}^{m, n}\left[\left.\left(\frac{a x^{k}}{\left(1+b x^{k}\right)}\right)^{s}\right|_{B^{*}} ^{A^{*}}\right] d x=$

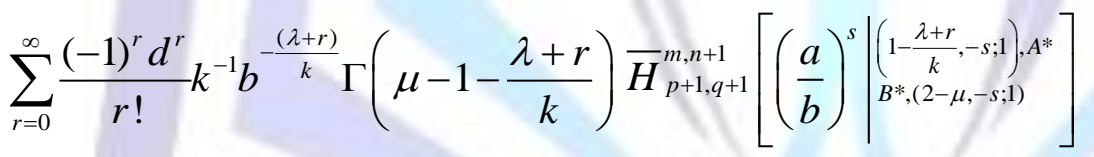

Where $\operatorname{Re}(d)>0, \operatorname{Re}\left(\frac{\lambda}{k}\right)<\operatorname{Re}(\mu-1), \operatorname{Re}\left(\lambda-k s \frac{b_{j}}{\beta_{j}}\right)>0, b>0$. The result follows by expanding $e^{-d x}$ and integrating term by term by applying result 1 .

Result 3

$\int_{0}^{\infty} x^{\lambda-1}\left(1-\frac{x}{y}\right)^{\mu-1} \bar{H}_{p, q}^{m, n}\left[\left.a x^{\nu}\right|_{B^{*}} ^{A^{*}}\right] d x=y^{\lambda} \Gamma(\mu) \bar{H}_{p+1, q+1}^{m, n+1}\left[\left.\left(a y^{\nu}\right)\right|_{B^{*},(1-\lambda-\mu,-v ; 1)} ^{(1-\lambda, v 1), A^{*}}\right]$

Where $\operatorname{Re}\left(\lambda-v \frac{b_{j}}{\beta_{j}}\right)>0$ for $j=1,2, \ldots, m ; \operatorname{Re}(\mu)>0$.

\section{A General Probability Function}

Here, we introduce a general probability density function $P(x)$ by using the most generalized function, namely the $\bar{H}$. function. Such a generalized form is not necessary to obtain all the classical central and non-central distributions as special cases form this general distribution. Special cases which can be expressed in more compact form are given later. Without any loss of generality the function $P(x)$ is assumed to be non-negative since the parameters can always be 
chosen in such a way that $P(x)$ is always non-negative and still several parameters will be left to our choice so that several classes of non-negative functions can be obtained as special cases and the general nature of $P(x)$ is not lost either.

$$
P(x)=\frac{e^{-d x} \frac{x^{\lambda-1}}{\left(1+b x^{k}\right)^{\mu-1}} \bar{H}_{p, q}^{m, n}\left[\left(\frac{a x^{k}}{\left(1+b x^{k}\right)}\right)^{s}||_{B^{*}}^{A^{*}}\right]}{C(d)}
$$

For $x>0$ and $P(x)=0$ elsewhere, where

$$
C(d)=\sum_{r=0}^{\infty} \frac{(-1)^{r} d^{r}}{r !} k^{-1} b^{-\frac{(\lambda+r)}{k}} \Gamma\left(\mu-1-\frac{\lambda+r}{k}\right) \bar{H}_{p+1, q+1}^{m, n+1}\left[\left(\frac{a}{b}\right)^{s} \mid\left(\begin{array}{l}
\left(1-\frac{\lambda+r}{k},-s ; 1\right), A^{*} \\
B^{*},(2-\mu,-s ; 1)
\end{array}\right]\right.
$$

It should be pointed out the factor $x^{\mu-1}\left(1+b x^{k}\right)^{\mu-1}$ can be absorbed inside the $\bar{H}$-function but it is written outside for convenience of manipulation later and when $d=0, C(d)$ can be written in a simple compact form as,

$C(0)=k^{-1} b^{-\frac{\lambda}{k}} \Gamma\left(\mu-1-\frac{\lambda}{k}\right) \bar{H}_{p+1, q+1}^{m, n+1}\left[\left(\frac{a}{b}\right)^{s} \mid \begin{array}{l}\left(1-\frac{\lambda}{k},-s ; 1\right), A^{*} \\ B^{*},(2-\mu,-s ; 1)\end{array}\right]$

Then the probability function $P(x)$ reduces to

$$
q(x)=\frac{\frac{x^{\lambda-1}}{\left(1+b x^{k}\right)^{\mu-1}} \bar{H}_{p, q}^{m, n}\left[\left(\frac{a x^{k}}{\left(1+b x^{k}\right)}\right)^{s} \mid{ }^{A^{*}}\right]}{C(0)}
$$

Almost all the classical central and non-central distributions can be obtained from $q(x)$ which will be seen later. In order to obtain all the useful classical central and non-central distributions as special cases it is not necessary to take general density function in the form of $P(x)$. In the light of the result 2 and 3 of section 2 it is easily seen that

$$
\int_{0}^{\infty} P(x) d x=1
$$

\section{Special Cases}

If we put $A_{j}=B_{j}=1$ in (2.1), (2.3) and (2.4), we get the result given by Mathai and Saxena [11] with a little simplification as:

$$
\begin{aligned}
& \int_{0}^{\infty} \frac{x^{\lambda-1}}{\left(1+b x^{k}\right)^{\mu-1}} H_{p, q}^{m, n}\left[\left(\frac{a x^{k}}{\left(1+b x^{k}\right)}\right)^{s} \mid \begin{array}{l}
\left(a_{j}, \alpha_{j}\right)_{1, p} \\
\left(b_{j}, \beta_{j}\right)_{1, q}
\end{array}\right] d x= \\
& k^{-1} b^{-\frac{\lambda}{k}} \Gamma\left(\mu-1-\frac{\lambda}{k}\right) H_{p+1, q+1}^{m, n+1}\left[\left(\frac{a}{b}\right)^{s} \mid \begin{array}{l}
\left(1-\frac{\lambda}{k},-s\right),\left(a_{j}, \alpha_{j}\right)_{1, p} \\
\left(b_{j}, \beta_{j}\right)_{1, q},(2-\mu,-s)
\end{array}\right]
\end{aligned}
$$

With the conditions given in (2.1).

$$
\int_{0}^{\infty} e^{-d x} \frac{x^{\lambda-1}}{\left(1+b x^{k}\right)^{\mu-1}} H_{p, q}^{m, n}\left[\left(\frac{a x^{k}}{\left(1+b x^{k}\right)}\right)^{s} \mid \begin{array}{l}
\left(a_{j}, \alpha_{j}\right)_{1, p} \\
\left(b_{j}, \beta_{j}\right)_{1, q}
\end{array}\right] d x=
$$




$$
\sum_{r=0}^{\infty} \frac{(-1)^{r} d^{r}}{r !} k^{-1} b^{-\frac{(\lambda+r)}{k}} \Gamma\left(\mu-1-\frac{\lambda+r}{k}\right) H_{p+1, q+1}^{m, n+1}\left[\left(\frac{a}{b}\right)^{s} \mid \begin{array}{l}
\left(1-\frac{\lambda+r}{k},-s\right),\left(a_{j}, \alpha_{j}\right)_{1, p} \\
\left(b_{j}, \beta_{j}\right)_{1, q},(2-\mu,-s)
\end{array}\right]
$$

With the conditions given in (2.3).

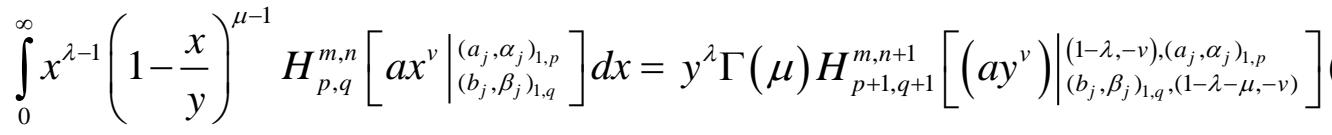

With the conditions given in (2.4).

Non-central Chi-square Distribution: The density function for the non-central chi-square is given by

$$
m(x)=e_{0,}^{-\frac{\mu^{2}}{2 \sigma^{2}} \sum_{r=0}^{\infty} \frac{1}{r ! \Gamma\left(r+\frac{k}{2}\right)}\left(\frac{\mu^{2}}{2 \sigma^{2}}\right)^{r}\left(\frac{1}{2}\right)^{\left(r+\frac{k}{2}\right)} e^{-\frac{1}{2} x} x^{r+k-1} \text { for } 0<x<\infty}
$$

Put $d=\frac{1}{2}, \mu=1, \lambda=\frac{k}{2}, s=k=1, b=0, b_{1}=0, b_{2}=1-\frac{k}{2}, \beta_{1}=\beta_{2}=1, a=\frac{\mu^{2}}{4 \sigma^{2}}, A_{j}=1=B_{j}$ in $P(x)$.Using the formula,

$$
\Gamma\left(\frac{k}{2}\right) G_{0,2}^{1,0}\left(-\frac{\mu^{2}}{4 \sigma^{2}} \mid\left(0,1-\frac{k}{2}\right)\right)={ }_{0} F_{1}\left(\frac{k}{2} ; \frac{\mu^{2} x}{4 \sigma^{2}}\right)
$$

And letting $b \rightarrow 0, P(x)$ reduces to $m(x)$ after a little simplification. In order to obtain the non-central $F$, non-central Beta, Student- $t$ and a number of classical central distributions we need consider only $q(x)$ or $P(x)$ when $d=0$ and it may be noticed that $q(x)$ is in a compact form.

Non-central $F$ Distribution : The density function for non-central $F$ is given by

$g(x)={ }_{0}$ elsewhere

$$
-\frac{\lambda^{2}}{2} \sum_{m=0}^{\infty}\left(\frac{\lambda^{2}}{2}\right)^{r}\left(\frac{1}{r !}\right) \frac{\Gamma\left(\frac{k+m}{2}+r\right)}{\Gamma\left(\frac{k}{2}+r\right) \Gamma\left(\frac{m}{2}\right)} \frac{x^{r+\frac{k}{2}-1}}{(1+x)^{r+\frac{+}{2}}} \text { for } x>0
$$

By putting $d=0, \mu=\frac{k+m}{2}+1, \lambda=\frac{k}{2}, s=k=1, b=1, b_{1}=1, b_{2}=1-\frac{k}{2}, \beta_{1}=\beta_{2}=1, a_{1}=1-\frac{k+m}{2}$,

$A_{j}=1=B_{j}, \alpha_{1}=\beta_{1}=\beta_{2}=1, m=n=p=1, q=2$, the $\bar{H}$-function reduces to the $G$-function of the desired form here. Then by using the general properties that,

$G_{1,2}^{1,1}\left(\left.x\right|_{(0,1-c)} ^{(1-a)}\right)=\frac{\Gamma(a)_{1} F_{1}(a ; z ;-x)}{\Gamma(c)}$ and $G_{0,1}^{1,0}(x \mid 0)=e^{-x}$

$P(x)$ Reduces to $g(x)$. By a simple change of variables we get the non-central Beta distribution, with the density function,

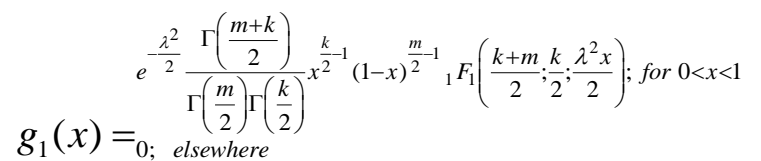

It may be noted that the conditional distribution of the multiple correlation coefficient under the condition of given values of the observations on the variables in a multivariable normal case, see ([12],p.509), is a non-central Beta distribution. 
Non-central Student- $t$ Distribution: The density function for the non-central Student- $t$ distribution is given by:

$$
h(x)=\frac{v^{\frac{v}{2}} e^{-\frac{\delta^{2}}{2}} \sum_{r=0}^{\infty} \Gamma\left(\frac{v+1+r}{2}\right)\left(\frac{\delta^{2}}{r !}\right)\left(\frac{2 x^{2}}{v+x^{2}}\right)^{\frac{r}{2}}}{\Gamma\left(\frac{v}{2}\right)\left(v+x^{2}\right)^{\frac{v+1}{2}}} ;-\infty<x<\infty
$$

Where $\delta$ is the non-centrality parameter and $k$ is the degrees of freedom. For convenience we will take the distribution in the folded form, that is

$$
\begin{aligned}
h_{1}(x) & =2 h(x) \text { for } x>0 \\
& =0, \text { elsewhere }
\end{aligned}
$$

Put $d=0, \mu=\frac{v+3}{2}+1, \lambda=1, b=1, b_{1}=0, \beta_{1}=1, a_{1}=\frac{1-v}{2}, A_{j}=1=B_{j}, \alpha_{1}=\frac{1}{2}, m=n=p=q=1, k=2$, replace $b$ by $\frac{1}{v}$ and $a$ by $\frac{2 \delta^{2}}{v}$. Then $P(x)$ reduces to $h_{1}(x)$.

The generalized hypergeometric function: The authors in [] introduced a general probability distribution from where the following distributions were obtained as special cases: the general hypergeometric distribution, the generalized gamma, gamma, generalized $F, F^{\prime}$, Student- $t$, Beta, Exponential, Cauchy, Weibull, Raleigh, Waiting time and logistic. The density function employed was,

$$
f(x)=\frac{\Gamma a^{\frac{c}{e}} \Gamma(\alpha) \Gamma(\beta) \Gamma\left(\frac{\gamma-c}{e}\right) x^{c-1}}{\Gamma\left(\frac{c}{e}\right) \Gamma\left(\frac{\alpha-c}{e}\right) \Gamma\left(\frac{\beta-c}{e}\right) \Gamma(\gamma)}{ }_{0} F_{1}\left(\alpha, \beta ; \gamma ;-a x^{e}\right) ; \text { for } x, c>0, \frac{\alpha-c}{e}>0, \frac{\beta-c}{e}>0
$$

This can be obtained as a special case from $P(x)$ by making the following substitutions. Put

$\lambda=c, d=0, b=0, s=1, a_{1}=1-\alpha, a_{2}=1-\beta, b_{1}=0, b_{2}=1-\gamma, k=c, \alpha_{1}=\alpha_{2}=\beta_{1}=\beta_{2}=1$

$A_{j}=1=B_{j}$. Using the formula

$H_{2,2}^{1,2}\left[\left.x\right|_{(0,1),(1-c, 1)} ^{(1-a, 1),(1-b, 1)}\right]=\frac{\Gamma(a) \Gamma(b)}{\Gamma(c)}{ }_{2} F_{1}(a, b ; c ;-x)$

We get $f(x)$ from $P(x)$ after a little simplification.

The Ratio Distribution: The ratio distribution can be obtained as:

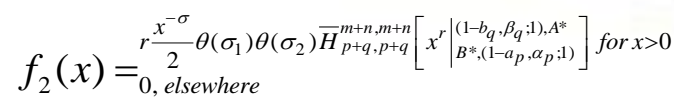

Where

$$
\theta\left(\sigma_{j}\right)=\frac{\prod_{k=m+1}^{q}\left\{\Gamma\left(1-b_{k}-\beta_{k} \frac{\sigma_{j}}{r}\right)\right\}^{B_{j}} \prod_{k=n+1}^{p} \Gamma\left(a_{k}+\alpha_{k} \frac{\sigma_{j}}{r}\right)}{\prod_{k=1}^{n}\left\{\Gamma\left(1-a_{k}-\alpha_{k} \frac{\sigma_{j}}{r}\right)\right\}^{A_{j}} \prod_{k=n+1}^{p} \Gamma\left(b_{k}+\beta_{k} \frac{\sigma_{j}}{r}\right)}
$$


From the structure of $f_{2}(x)$ itself it is evident that $f_{2}(x)$ can be obtained from $P(x)$ by making suitable changes in the parameters. Thus $P(x)$ also contains the density function of the ratio of two independent stochastic variables whose density functions can be expressed in terms of any known special function.

\section{The Characteristic Function and Moments}

Since the characteristic function is defined as

$$
\underline{\theta}(t)=E\left(e^{i t x}\right)=\int_{0}^{\infty} e^{i t x} P(x) d x
$$

Where $i=\sqrt{(-1)}$, it can be easily obtained by replacing the parameter $d$ by $d-i t$ and hence

$$
\underline{\theta}(t)=\frac{C(d-i t)}{C(d)}
$$

Where $C(d)$ is given in (3.2). Hence the moments and cumulates can be evaluated without much difficulty.

Moments: The $v^{\text {th }}$ moment about the origin, $M_{v}$ is obtained by replacing $\lambda$ by $\lambda+v$ in (3.1) and then taking the ratio of the normalizing factors in $P(x)$. That is

$$
M_{v}=\frac{C(d, \lambda+v)}{C(d, \lambda)}
$$

Where

$$
C(d, \lambda)=\sum_{r=0}^{\infty} \frac{(-1)^{r}}{r !} d^{r} k^{-1} b^{-\frac{\lambda+r}{k}} \Gamma\left(-1+\mu-\frac{\lambda+r}{k}\right) \bar{H}_{p+1, q+1}^{m, n+1}\left[\left(\frac{a}{b}\right)^{s} \mid\left(\begin{array}{l}
\left.1-\frac{\lambda+r}{k}, s ; 1\right), A^{*} \\
B^{*},(2-\mu, s ; 1)
\end{array}\right]\right.
$$

And if $d=0$, this reduces to $\frac{C(0, \lambda+r)}{C(0, \lambda)}$, where

$C(0, \lambda)=k^{-1} b^{-\frac{\lambda}{k}} \Gamma\left(-1+\mu-\frac{\lambda}{k}\right) \bar{H}_{p+1, q+1}^{m, n+1}\left[\left(\frac{a}{b}\right)^{s} \mid \begin{array}{c}\left(1-\frac{\lambda}{k}, s ; 1\right), A^{*} \\ B^{*},(2-\mu, s ; 1)\end{array}\right]$

A Recurrence Relationship: A recurrence relationship among $M_{v}, M_{v-1}$ and $M_{v+1}$ can be obtained by using the recurrence relationships for the $\bar{H}$-function.

$$
M_{\mu, v}=\frac{1}{C(d)} \sum_{r=0}^{\infty} \frac{(-1)^{r}}{r !} d^{r} k^{-1} b^{-\frac{\lambda+r+v}{k}} \Gamma\left(-1+\mu-\frac{\lambda+r+v}{k}\right) \quad \bar{H}_{p+1, q+1}^{m, n+1}\left[\left(\frac{a}{b}\right)^{s} \mid\left(\begin{array}{l}
\left.1-\frac{\lambda+r+v}{k}, s ; 1\right), A^{*} \\
B^{*},(2-\mu, s ; 1)
\end{array}\right]\right.
$$

Where $C(d)$ is given in (3.2). On applying the recurrence formula for the $\bar{H}$-function.

$$
\begin{aligned}
& \left(1-a_{1}+b_{q}\right) \bar{H}_{P, Q}^{M, N}\left[x \mid \begin{array}{l}
\left(a_{j} ; \alpha_{j} ; A_{j}\right)_{1, N},\left(a_{j} ; \alpha_{j}\right)_{N+1, P} \\
\left(b_{j}, \beta_{j}\right)_{1, M},\left(b_{j}, \beta_{j} ; B_{j}\right)_{M+1, Q-1},\left(b_{Q}, \alpha_{1} ; B_{Q}\right)
\end{array}\right]= \\
& \bar{H}_{P, Q}^{M, N}\left[x \mid \begin{array}{l}
\left(a_{1}-1, \alpha_{1} ; A_{1}\right),\left(a_{j} ; \alpha_{j} ; A_{j}\right)_{2, N},\left(a_{j} ; \alpha_{j}\right)_{N+1, P} \\
\left.\left(b_{j}, \beta_{j}\right)_{1, M},\left(b_{j}, \beta_{j} ; B_{j}\right)_{M+1, Q-1}, b_{Q}, \alpha_{1} ; B_{Q}\right)
\end{array}\right]-\bar{H}_{P, Q}^{M, N}\left[x \mid \begin{array}{l}
\left(a_{j} ; \alpha_{j} ; A_{j}\right)_{1, N},\left(a_{j} ; \alpha_{j}\right)_{N+1, P} \\
\left(b_{j}, \beta_{j}\right)_{1, M},\left(b_{j}, \beta_{j} ; B_{j}\right)_{M+1, Q-1},\left(b_{Q}+1, \alpha_{1} ; B_{Q}\right)
\end{array}\right]
\end{aligned}
$$

To $M_{v}$ of (5.3), we see that $M_{v}$ is equal to

$$
\frac{1}{C(d)} \sum_{r=0}^{\infty} \frac{(-1)^{r}}{r !} d^{r} k^{-1} b^{-\frac{\lambda+r+v}{k}}\left(-2+\mu-\frac{\lambda+r+v}{k}\right) \Gamma\left(-2+\mu-\frac{\lambda+r+v}{k}\right)
$$




$$
\begin{aligned}
& \bar{H}_{p+1, q+1}^{m, n+1}\left[\left(\frac{a}{b}\right)^{s} \mid\left(\begin{array}{l}
\left.1-\frac{\lambda+r+v}{k}, s ; 1\right), A^{*} \\
B^{*},(2-\mu, s ; 1)
\end{array}\right]\right. \\
& =\frac{1}{C(d)} \sum_{r=0}^{\infty}(-1)^{r} d^{r} k^{-1} b^{-\frac{\lambda+r+v}{k}} \Gamma\left(-2+\mu-\frac{\lambda+r+v}{k}\right) \\
& \left\{\bar{H}_{p+1, q+1}^{m, n+1}\left[\left(\frac{a}{b}\right)^{s} \mid \begin{array}{l}
\left(1-\frac{\lambda+r+v}{k}, s ; 1\right), A^{*} \\
B^{*},(3-\mu, s ; 1)
\end{array}\right]-\bar{H}_{p+1, q+1}^{m, n+1}\left[\left(\frac{a}{b}\right)^{s} \mid \begin{array}{c}
\left(-\frac{\lambda+r+v}{k}, s ; 1\right), A^{*} \\
B^{*},(2-\mu, s ; 1)
\end{array}\right]\right\}
\end{aligned}
$$

Hence, we obtain

$$
M_{\mu, v}=M_{\mu-1, v}-b M_{\mu, v+1}
$$

\section{The Distribution Function}

The distribution function or the emulative density function

$$
F(y)=\int_{0}^{y} P(x) d x
$$

Can be obtained foe some special forms of $P(x)$. By putting $s=1$ and taking the limit $d \rightarrow 0$ and $b \rightarrow 0, P(x)$ reduces to the form

$$
P_{1}(x)={ }_{0, \text { elsewhere }}{ }^{\frac{\lambda}{r}} \theta(\sigma) x^{\lambda-1} \bar{H}_{p, q}^{m, n}\left[a x^{v} \mid B^{*}\right] \text { for } x>0
$$

By using result (2.2), we get

$$
\int_{0}^{y} P_{1}(x) d x=r \theta^{\frac{\lambda}{r}} \theta(\lambda) y^{\lambda} \bar{H}_{p, q}^{m, n}\left[\left.a y^{v}\right|_{B^{*},(-\lambda, v ; 1)} ^{(1-\lambda, v 11), A^{*}}\right]
$$

Where $\operatorname{Re}\left(\lambda+v \frac{b_{j}}{\beta_{j}}\right)>0 ; j=1,2, \ldots, m$ and $\theta(\lambda)$ is defined in (4.13). By using $F(y)$ we can obtain the distributions of order statistics and other related statistics which we will not discuss here.

\section{References}

1. Boersma, J.; On a function which is a special case of Meijer's $G$-function, Compositio Mathematica, 15(1962), 34-63.

2. Braaksma, B.L.J.; Asymptotic expansions and analytic continuation for Barnes-Integrals, Compositio Mathematica,15(1964),239-241.

3. Buschman, R.G. and Srivastava, H.M. 1990. The $\bar{H}$ - function associated with a certain class of Feynman integrals, J.Phys.A:Math. Gen. 23, 4707-4710.

4. Erdelyi, A. et. al.; Higher Transcendental Functions, vol. I, McGraw-Hill, New York (1953).

5. Erdelyi, A. et.al.; Tables of Integral Transforms, vol. I, McGraw-Hill, New York (1954).

6. Fox, C. 1961.The $G$ and $H$-function as Fourier kernels. Trans. Amer. Math. Soc.98:395-429.

7. Gupta, K.C.; On $H$-functiona, Annales de la Societe Scientifique de Bruxelles, T. 79,II(1965), 97-106.

8. Inayat-Hussain, A.A.; New properties of hypergeometric series derivable from Feynman integrals :Il A generalization of the $H$-function, J. Phys. A. Math. Gen. 20(1987).

9. Mathai, A.M. and Saxena, R.K.; On a generalized hypergeometric distribution, Matrika, 11(1966), 127-132.

10. Mathai, A.M. and Saxena, R.K.; Tables of the generalized hypergeometric distribution, Matrika, 11(1966),163-169. 
11. Mathai, A.M. and Saxena, R.K.; A generalized probability Distribution,Sepatata de la revista mathematica Y Fisica Teorica, vol. 21 (1971),193-202.

12. Rao, C.R.; Linear Statistical Inference and Its Applications, Wiley, NewYork (1965).

13. Rathi, A.K. 1997. A new generalization of generalized hypergeometric functions. Le Mathematiche Fasc. II52:297310. 